\title{
Mediastinal mass: A diagnostic conundrum
}

\author{
Vamsidhar Rachapalli, MRCS, Anil John, FRCS, and Michael J. Unsworth-White, FRCS (CTh), Plymouth, United Kingdom
}

\section{Clinical Summary}

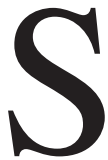

eptic arthritis of the sternoclavicular joint (SCJ) is a rare condition that can present as anterior mediastinal disease with venous obstruction and can be a clinical conundrum. ${ }^{1}$ We present one such case, which was resolved only after incision and drainage.

A 74-year-old man had a 2-week history of a tender mass situated at the suprasternal notch and extending over the right clavicle. It was associated with swelling of the right upper limb of similar duration. He also reported recent onset of shortness of breath. He had a history of ischemic heart disease for which he underwent coronary artery bypass grafting 8 years earlier. Other comorbidities included hypertension and atrial fibrillation.

On examination, he was apyrexic and had rate-controlled atrial fibrillation. He had a 3 by $4 \mathrm{~cm}$ fluctuant nonpulsatile right suprasternal swelling extending retrosternally. His right arm was swollen with pitting edema but with intact sensorimotor function.

Thoracic computed tomographic scan demonstrated a 7 by 6 $\mathrm{cm}$ soft tissue density in the right superoanterior aspect of the chest extending retrosternally to the confluence of the right jugular and subclavian arteries and right SCJ (Figure 1).

The mass was incised and pus drained the following day. The cavity extended into the right SCJ. Beta-hemolytic streptococcus was cultured from the pus and the patient was commenced on the appropriate antibiotics. The patient's condition gradually improved and the swelling of the right arm subsided.

\section{Discussion}

Septic arthritis of the SCJ is a rare condition, which was first reported in 1896 by Vogelieus, as recounted by Mohyuddin. ${ }^{1}$ The SCJ is said to be involved in $2 \%$ of cases of septic arthritis. ${ }^{2}$ Some authors have reported that it may account for as many as $9 \%$ of cases. ${ }^{3}$

The SCJ involvement is associated with diabetes, systemic steroid therapy, alcoholism, intravenous heroin abuse, immunoparesis, rheumatoid arthritis, and local infection and can occur as a rare complication of subclavian vein thrombosis. ${ }^{2,4}$ It has also been reported in healthy or apparently healthy individuals. ${ }^{4}$

From the Department of Cardiothoracic Surgery, Derriford Hospital, Plymouth, United Kingdom.

Received for publication July 25, 2006; accepted for publication Sept 5, 2006.

Address for reprints: V. Rachapalli, MRCS, 8, Ffordd Ty Unnos, Cardiff CF14 4NJ, United Kingdom (E-mail: r_vamsidhar@yahoo.com).

J Thorac Cardiovasc Surg 2007;133:586-7

$0022-5223 / \$ 32.00$

Copyright $\odot 2007$ by The American Association for Thoracic Surgery doi:10.1016/j.jtcvs.2006.09.048

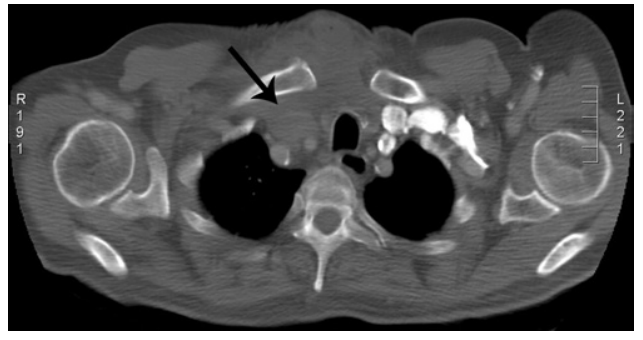

Figure 1. Computed tomographic scan. Retrosternal extension of the abscess (arrow) arising from the right supraclavicular joint.

Septic involvement of the SCJ presents with minimal symptoms, as it usually does not manifest as a swollen and painful joint. ${ }^{1}$ Most often, it presents as a complication, resembling other causes of anterior chest wall pain, such as tumors of the clavicle and sternum and even pneumonia. ${ }^{4}$ Complications include retrosternal abscess, fistula formation, mediastinitis, superior vena cava syndrome, and osteomyelitis. ${ }^{1}$ Mediastinal involvement can be life-threatening, especially in immunocompromised patients. ${ }^{2}$ Suspicion should be entertained when patients have a swelling over the joint. ${ }^{1} \mathrm{We}$ are reporting the first case in which it has presented as subclavian compression syndrome. Early recognition is of paramount importance to prevent more serious complications such as mediastinitis or superior vena cava syndrome. ${ }^{1}$

The organism most usually implicated is Staphylococcus aureus. ${ }^{4}$ Other organisms isolated include Brucella spp, Serratia marcescens, Candida albicans, and Streptococcus. Pseudomonal arthritis has been reported in subjects addicted to heroin and intravenous drugs. ${ }^{4}$

Computed tomography is the imaging modality of choice. It not only helps in demonstrating the anatomy of the joint and surrounding soft tissues, but it also aids aspiration of joint fluid in septic arthritis. ${ }^{2}$ Magnetic resonance imaging is used as an adjunct to further demonstrate joint involvement. ${ }^{2,4}$

SCJ septic arthritis can be managed conservatively and surgically. Carlos and associates ${ }^{5}$ recommend conservative management for early cases, but in the presence of extra-articular involvement, aggressive surgery with en bloc resection would result in eradication of infection with minimal disability.

Although rare, SCJ septic arthritis is associated with significant morbidity. Its diverse presentations can be a diagnostic challenge.

\section{References}

1. Mohyuddin A. Sternoclavicular joint septic arthritis manifesting as a neck abscess: a case report. Ear Nose Throat J. 2003;82:618-21.

2. LeLoet X, Vittecoq O. The sternocostoclavicular joint: normal and abnormal feature. Joint Bone Spine. 2002;69:161-9.

3. Yood RA, Goldenberg DL. Sternoclavicular joint arthritis. Arthritis Rheum. 1980;23:232-9. 
4. Gillis A, Friedman B, Caraco Y, Blankstein A, Yellin A, Friedman G. Septic arthritis of the sternoclavicular joint in healthy adults. $J$ Intern Med. 1990;228:275-8.
5. Carlos GN, Kesler KA, Coleman JJ, Broderick L, Turrentine MW, Brown JW. Aggressive surgical management of sternoclavicular joint infections. J Thorac Cardiovasc Surg. 1997;113:242-7.

\title{
Bone or pleura? Primary pleural osteosarcoma
}

\author{
Pankaj Chandak, MRCS(Eng), ${ }^{a}$ Ian Hunt, MRCS(Eng), ${ }^{a}$ Randolph Rawlins, FRCS, ${ }^{\text {a }}$ Sebastian Lucas, FRCPath, \\ and Tom Treasure, MD, MS, FRCS, ${ }^{a}$ London, United Kingdom
}

$\mathrm{W}$

e describe a case of primary pleural osteosarcoma presenting as recurrent bloody pleural effusion and striking pleural calcification encasing the lung.

\section{Clinical Summary}

A 76-year-old nonsmoking ex-Royal Navy engineer had a 2-month history of progressive breathlessness and recurrent right-sided bloody effusion. The chest computed tomographic scan showed striking pleural calcification of variable thickness encasing the right lung and a large pleural effusion (Figure 1, $A$ and $B$ ). We did not recognize the appearances. Our differential diagnosis included old trauma and tuberculosis, but there was no history of either. Immunohistochemistry of pleural needle biopsy specimens raised suspicion of malignant pleural mesothelioma. He came to us for surgical biopsy. We performed a short $(3-\mathrm{cm})$ rib resection and took a full-thickness pleural biopsy specimen through the bed of the rib. Not to our surprise, pleurodesis was ineffective.

The pleural specimen was so bony that the pathologist initially mistook it for rib. On histologic review the diagnosis of primary pleural osteosarcoma was made (Figure 2). He was referred for specialist oncologic management.

\section{Discussion}

Calcification of the pleura is a sequela of intrapleural hemorrhage, infection, tuberculosis, or asbestos-related plaques. Extraskeletal osteosarcoma is a rare malignant mesenchymal neoplasm characterized by production of osteoid. It represents $1 \%$ to $2 \%$ of all soft tissue sarcomas and $4 \%$ of all osteosarcomas. ${ }^{1}$ Since first described by Wilson ${ }^{2}$ in 1941 , a few series of these tumors have been reported. To qualify as an extraskeletal osteosarcoma, a tumor must arise in soft tissue, have a uniform sarcomatous pattern, and

From the Departments of Thoracic Surgerya and Histopathology, ${ }^{\mathrm{b}}$ Guy's \& St Thomas's Hospital, London, United Kingdom.

Received for publication July 25, 2006; accepted for publication Sept 5, 2006.

Address for reprints: Ian Hunt, MRCS(Eng), Department of Thoracic Surgery, Guy's Hospital, St Thomas Street, London SE1 9RT, United Kingdom (E-mail: ianjhunt@gmail.com).

J Thorac Cardiovasc Surg 2007;133:587-8

$0022-5223 / \$ 32.00$

Copyright $\odot 2007$ by The American Association for Thoracic Surgery doi:10.1016/j.jtcvs.2006.09.050 produce osteoid. ${ }^{3}$ Men are slightly more affected and the peak prevalence occurs in the sixth decade. Symptoms may relate to a slow-growing mass. Common sites of disease include the soft tissues of the extremities or the retroperitoneum, although a variety of pleural cavity sarcomas have been described, including liposarcomas, chondrosarcomas, and osteosarcomas. ${ }^{4}$

Although pathogenesis of extraskeletal osteosarcoma remains unclear, several possibilities have been suggested. These include trauma with subsequent myositis ossificans, radiation, metaplasia of connective tissue, and malignant degeneration of embryonal somatic remnants. ${ }^{5}$
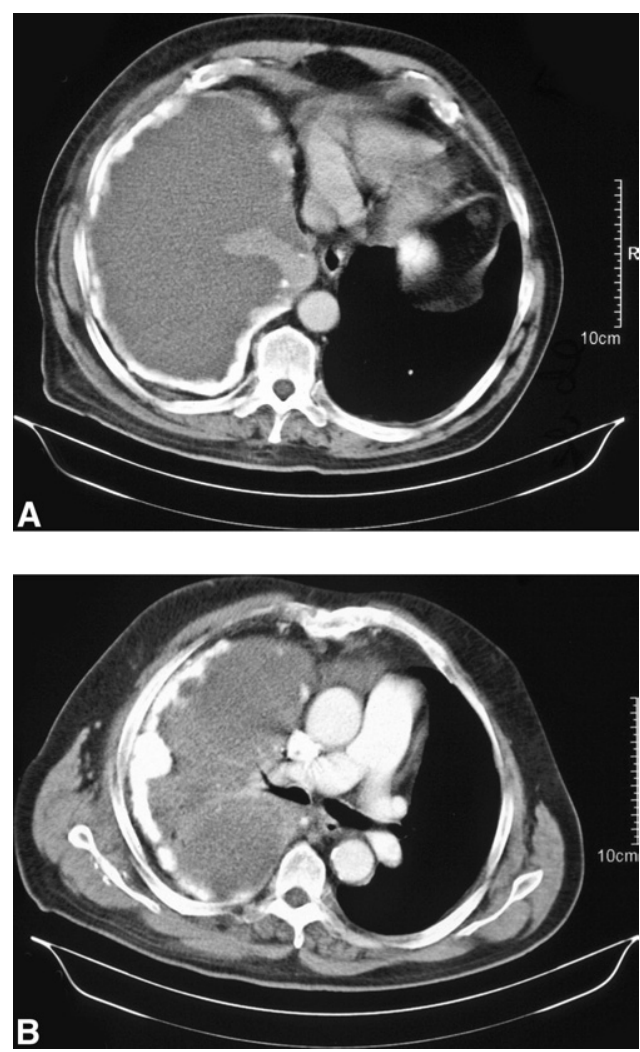

Figure 1. A and B, Chest computed tomographic scans showed striking pleural calcification of variable thickness encasing the right lung and a large pleural effusion, but no significant contraction of the hemithorax. There were no obvious intrathoracic mass lesions. 